\title{
Spatial Pyramid Deep Hashing for Large-Scale Image Retrieval
}

\author{
Wanqing Zhao ${ }^{\mathrm{a}}$, Hangzai Luo ${ }^{\mathrm{a}, *}$, Jinye Peng ${ }^{\mathrm{a}}$, Jianping Fan ${ }^{\mathrm{b}}$ \\ ${ }^{a}$ School of information and technology, Northwestern University, Shaanxi 710127, China \\ ${ }^{b}$ Department of Computer Science, UNC-Charlotte, NC28223, USA
}

\begin{abstract}
Effective feature representations and similarity measurements are crucial for large-scale image retrieval, and conventional methods often learn hash functions from a predefined hand-crafted feature space. Meanwhile, the spatial structure in raw images always lost in most previous methods. Encouraged by the recent advances in convolutional neural networks(CNNs), a novel Spatial Pyramid Deep Hashing(SPDH) algorithm is developed for the task of fast image retrieval. In our SPDH algorithm, the CNN with a spatial pyramid pooling and a locally-connected layer with binary activation functions is utilized to build the end-to-end relation between the raw image data and the binary hashing codes for fast indexing. Different from the fully-connected layer, the locally-connected layer can consider each local spatial bin as an independent unit and only connect the local bin to preserve the spatial pyramid structure for hash codes. The learning of both the hash function and the feature representations are jointly optimized via backward propagation with classification or similarity loss function on the large-scale labeled dataset such as ImageNet. Moreover, a spatial pyramid binary pattern matching algorithm is developed to achieve partial local similar matching among the images. Our experimental results have shown that our SPDH method can outperform several state-of-the-art hashing algorithms on the CIFAR-10, SIVAL and the Oxford buildings datasets.
\end{abstract}

Keywords: Image Retrieval, Hashing Learning, Convolutional Neural Networks, Spatial Pyramid Structure

${ }^{*}$ Corresponding author: hzluo@nwu.edu.cn

Preprint submitted to Neurocomputing

January 19, 2017

(C) 2016. This manuscript version is made available under the Elsevier user license http://www.elsevier.com/open-access/userlicense/1.0/ 


\section{Introduction}

Content-based image retrieval (CBIR) has extensively been studied by the community of multimedia computing for decades, but the performance of most existing CBIR systems crucially depends on the effectiveness of the underlying techniques for image characterization [1][2][3] and similarity measurement. Although a variety of techniques have been proposed to resolve this problem, it still remains as one of the most challenging problems in current CBIR research area because of the well-known "semantic gap" between low-level image pixels captured by machines and high-level semantic concepts perceived by human. Recent studies have shown that the deep features from convolutional neural networks(CNNs) can significantly improve the performance of various computer vision tasks, such as image classification, object detection and segmentation [4][5][6][7]. These accomplishments are attributed to the deep CNNs attempting to model high-level abstractions in images by employing deep architectures composed of multiple non-linear transformations. However, the prevalent CNNs require a fixed size of input images. Such a predefined scale may not be suitable for large-scale image retrieval when an object scales abruptly in the query image. Besides, feature representations learned from $\mathrm{CNNs}$ are usually in high-dimensions that are inefficient for large-scale image retrieval application.

Due to its fast speed for query processing and low cost for storage, hashing has successfully been used for large-scale image retrieval. Traditional hashing learning algorithms for image retrieval usually include two sequential steps. First, all images of interest are encoded to feature vectors by hand-crafted descriptors. One of the most popular approaches is to extract local descriptors and represent an image in bag-of-features (BoF) [8] or Sparse coding [9][10]. Second, feature vectors are mapped into a low-dimensional real-valued space by using machine learning techniques and further converted to binary codes by some approaches, such as thresholding or pair-wised methods. However, the above two steps are generally treated as two independent issues, results in decreased performance.

On the other hand, unlike text documents which are usually represented by bag of words, images exist strong spatial structure and geometric constraints [11][12]. Figure 1 shows some examples where the duplicate objects are a part of the whole images, and these images can easily be judged from the same building or trademarks by human. However, most existing hashing learning approaches ignored the spatial information of the features and the 

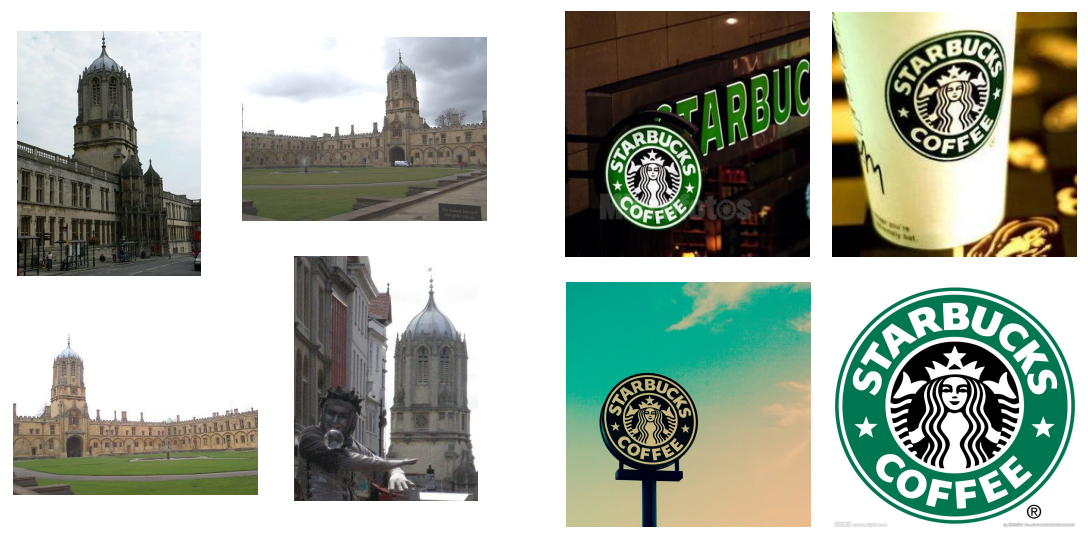

Figure 1: Examples of partially-similar images, where the similar region occupies a small portion of each image.

generated compact hash codes may weaken the ability on identifying partial similarity among the images, meanwhile supporting partial image matching is desirable for many retrieval applications.

In order to address the above mentioned issues, a novel Spatial Pyramid Deep Hashing(SPDH) algorithm is developed in this work for the task of large-scale image retrieval. The CNN with a spatial pyramid pooling is employed to develop the end-to-end solution between the raw image data and the binary hashing codes. By using the Spatial Pyramid Pooling (SPP) [13], we not only eliminate the restriction of fixed input image size in CNN, but also capture the spatial structure from raw image. We also employed some hidden layer with binary activation functions for the hashing codes and preserved the spatial pyramid structure. In our algorithm, both learning the hash function and learning the deep features are jointly optimized via backward propagation with classification or similarity loss function on the large-scale labeled dataset such as ImageNet. Moreover, a spatial pyramid binary pattern matching algorithm is developed to enable partial similarity matching among the images, which may significantly improve the accuracy rate for image retrieval.

This paper has made three main contributions for large-scale image retrieval application: (a) First, a deep learning method is developed for largescale image retrieval. It unifies the learning of deep features and the learn- 
ing of hash function via deep convolutional neural network with the spatial pyramid pooling layer and the binary hashing layer. (b) Second, our hashing method can preserve the spatial pyramid structure form raw images. By using our local pyramid hash matching algorithm, the flexibility and accuracy of large-scale image retrieval can be improved significantly. (c) Third, our method can learn binary hashing codes in a point-wised mode and is easily scalable to the data size in comparison of conventional pair-wised approaches. In our experiments on the public dataset CIFAR-10, SIVAL and Oxford buildings, our proposed method can well capture the partial similarity and outperform state-of-the-art hashing learning approaches.

The rest of the paper is organized as follows: the related works on image retrieval using hash learning are discussed in Section 2. In Section 3, we introduce our hashing learning algorithm and pyramid hash matching algorithm. The experimental results for algorithm evaluation are reported in Section 4, and we have compared our algorithm with multiple state-of-the-art methods. Finally we conclude our paper in Section 5.

\section{Related Works}

The existing image retrieval methods generally can be categorized into two types: (a) exact similarity retrieval methods; and (b) approximate similarity retrieval methods. Exact similarity retrieval methods can be implemented efficiently by using pre-built space-partitioning index structures (such as KD-tree) or data-partitioning index structures (such as R-tree). However, although some researchers [14] made kd-trees available for approximate nearest neighbor search, tree-based methods often lead their complexity to a linear scan in the worst case. In such a scenario, hash based methods can dramatically speed up high-dimensional approximate similarity search into virtually $\mathrm{O}(1)$ time complexity.

Such hash-based methods for image retrieval can be considered as encoding high-dimensional feature vectors to a low-dimensional Hamming space, while retaining as much semantic similarity structure of data as possible. The well-known hashing techniques preserving similarity information are Locality-Sensitive Hashing (LSH) [15] and its variants [16], and the Min-Hash algorithms [17]. These methods employ random hash functions to map data points to similar hash codes without any training. They usually scatter the hash codes in order to improve the accuracy of the matching. However, since 
the design of hash functions for these hash methods is data-independent, it may lead to quite inefficient (long) codes in practice.

Several recently proposed hashing techniques attempt to overcome this problem by finding good data-dependent hash functions through machine learning. The data-dependent hashes focus on how to learn compact hashing codes from the training data. These learning-based hashing methods usually consist of two phases: (1) encoding the high-dimensional features into lower dimensional space; and (2) quantizing the generated real-valued representations into binary hash codes. Existing data-dependent hash methods include unsupervised method [18], supervised method [19][20] and semi-supervised method [21]. Specifically, the unsupervised methods learn the hash functions by using unlabeled data, which encode neighborhood relation of samples from a certain metric space into the Hamming space. For example, to encode training data into binary codes, $\mathrm{SpH}$ tries to preserve the global similarity structures defined in the original space. For semi-supervised and supervised methods [22], the more abundant similarity information of training data is utilized to improve the hashing learning.

Compared to hand-crafted features, high-level abstractions captured by deep learning techniques have shown great potential in various visual recognition tasks such as object detection and image classification [4][5][6][23]. Krizhevsky et al. [4] achieve breakthrough performance on ImageNet by using seven layers of CNNs. Deep architectures also have been used for hash learning. Xia et al. [24] propose a supervised hashing method to learn binary hashing codes by using CNN and show state-of-the-art retrieval performance on public datasets. However, it requires the input of a pair-wised similarity matrix of the data and the similarity matrix is difficult to build on a large-scale dataset. Very recently, Lai et al. [25] proposed a simultaneous feature learning and hash coding framework based on CNN, and used a triplet ranking loss for preserving relative similarities. Another related work was proposed by Lin et al. [26], which learns hash codes and image representations in a point-wised manner. Pre-training on the large-scale image classification database (i.e., ImageNet) was used in this model.

\section{Spatial Pyramid Deep Hashing}

Instead of learning hash function on the hand-crafted features, the objec-

tive of this method is to learn a deep network that can project raw images of arbitrary sizes into $q$ dimensional binary code $h \in\{-1,1\}^{q}$, while preserving 


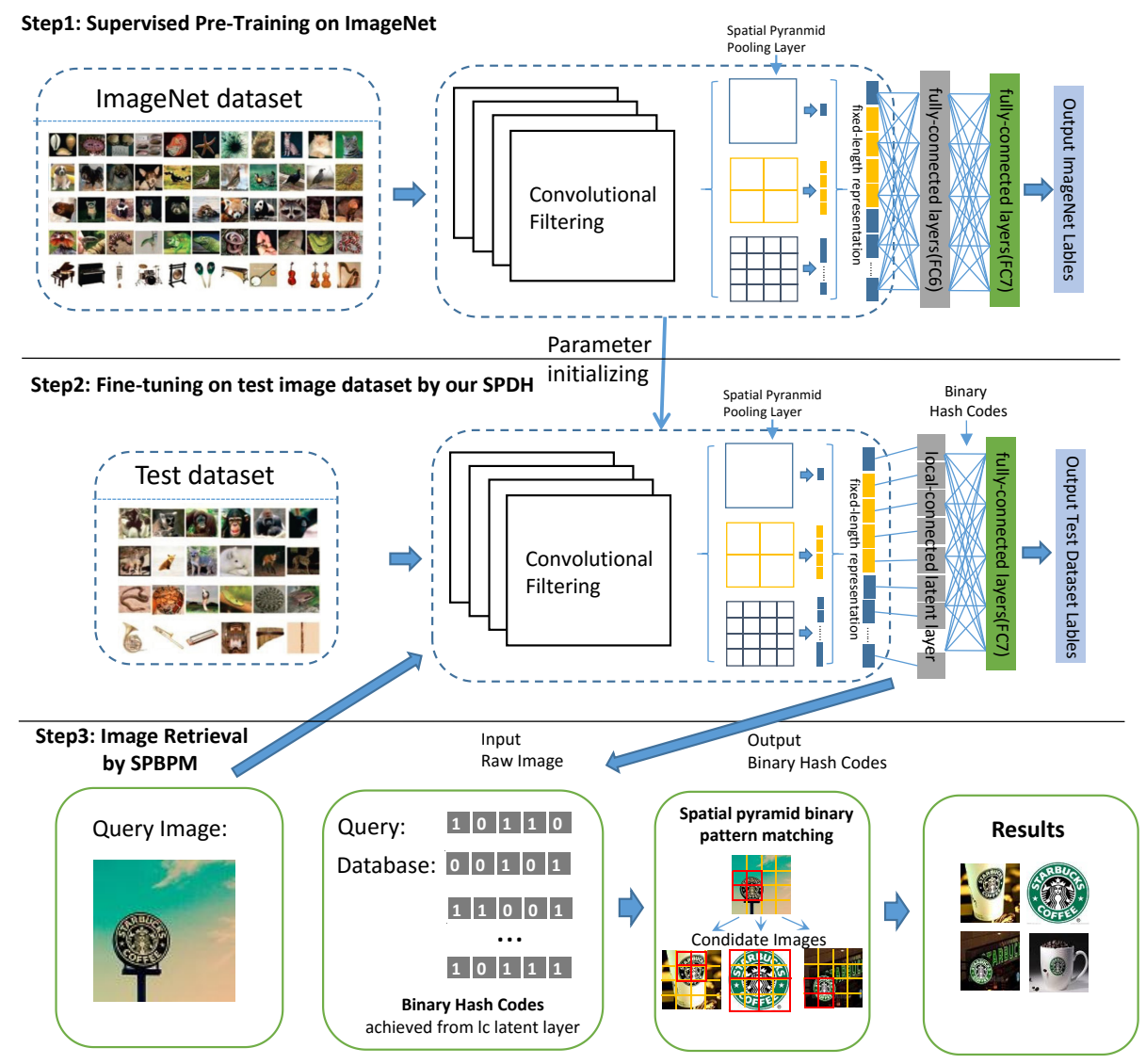

Figure 2: The proposed Spatial Pyramid Deep Hashing algorithm. 
the spatial pyramid structure and semantic consistency of each image. In this section, we introduce Spatial Pyramid Deep Hashing algorithms shown in Figure 2. The framework includes three main steps: (a) The first step is supervised pre-training on the large-scale ImageNet dataset [27] by using the model of SPP-net [13]. (b) The second step is to fine-tune the network with the locally-connected layer to learn domain specific feature representation and a set of hash-like function at the same time. (c) The third step is to retrieve the images similar to the query by using the proposed spatial pyramid binary pattern matching algorithm (SPBPM). This approach for learning binary codes and image retrieval by SPBPM is described in detail as follows.

\subsection{Learning Binary Codes with SPP-net}

Although CNNs have achieved promising results for many classification tasks, they are still difficult to be applied directly for image retrieval. First, image representation is always high-dimensional vectors that are inefficient for large-scale image retrieval. Second, the conventional CNNs require fixedsize (e.g. $224 * 224$ ) input images. But query images always have variable sizes and arbitrary resizing the query image may cause scale confusion. To facilitate efficient image retrieval, a practical way is to convert the feature vectors to binary codes. In order to incorporate the learning of deep CNN features for image representation and the learning of binary hash codes by an end-to-end learning framework with arbitrary size of raw images, we introduce the spatial pyramid pooling networks (SPP-nets) [13] into the process for hash code learning. The SPP-nets add a SPP layer on the top of the last convolutional layer. The SPP layer pools the features with spatial pyramid structure and removes the fixed-size limitation of prevalent CNN models. And it pools the responses of each filter for each spatial bin and the whole vectors are the input to the fully-connected layer $F_{7}$. Different from the SPP-net model, we need that the output binary features with spatial pyramid structure for final classification layer $F_{8}$. To fulfill this idea, we replace the $F_{7}$ with the locally-connected layer as show in the Figure 2. Different from the fully-connected layer, the locally-connected layer will consider each local spatial bin as an independent unit and only connect the local bin from the previous spatial pyramid pooling layer. Simultaneously, the neurons in the locally-connected layer are activated by sigmoid functions that approximate the activation to $\{0,1\}$ and the gradient can be computed by traditional

methods. Approximate binary feature representation will be the input for 


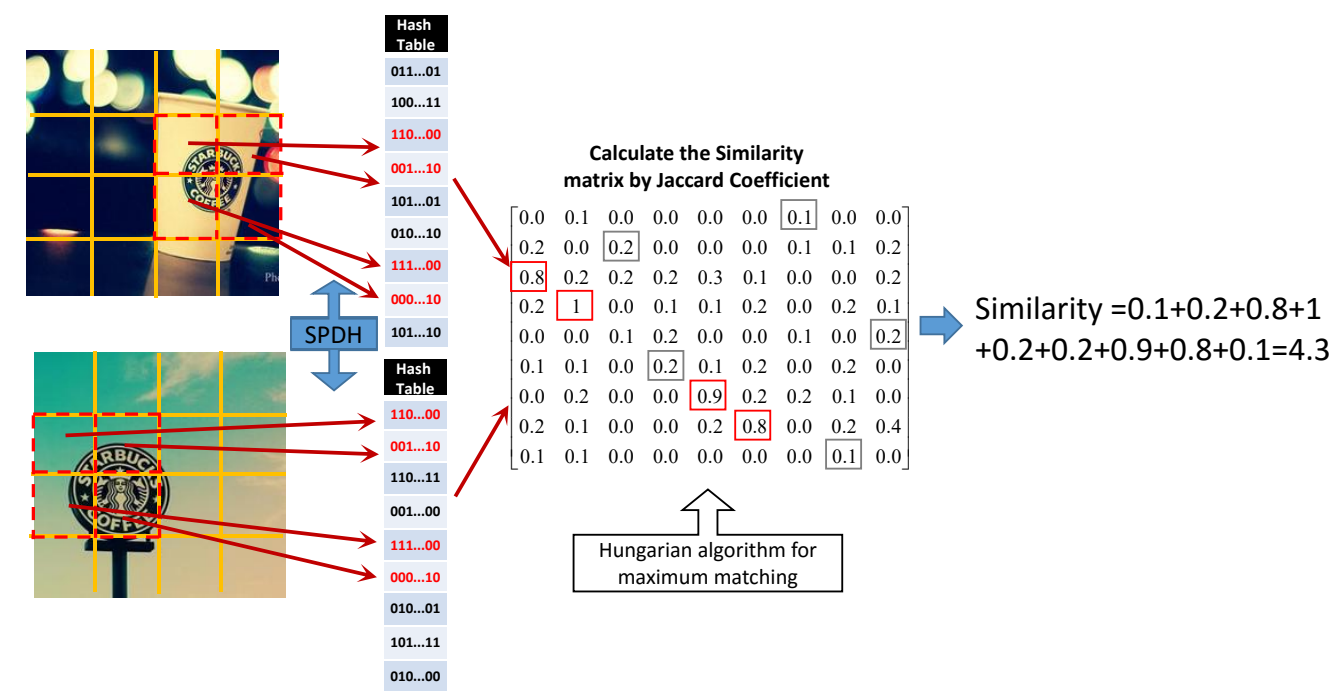

Figure 3: The illustration of our spatial pyramid binary pattern matching algorithm for similarity calculation and matching by using SPDH.

final classification layer $F_{8}$. In other words, images having similar binary codes would have the same label or semantics. Proposed locally-connected layer not only achieved binary feature abstraction with the spatial pyramid structure, but also inherited the semantic concept from label.

Next, we introduce how to optimize the network parameter given a set of training images. As in previous works, we first initial the weights of the deep $\mathrm{CNN}$ and the spatial pyramid pooling layer by using the training method introduced in SPP-net [13]. Then we fine-tune the proposed framework on different task database by initializing the weights of the locally-connected layer and the final classification layer $F_{8}$ randomly. The network structure can be trained with standard back-propagation from the Caffe or ConvNet CNN library.

\subsection{Image Retrieval by Partial Pyramid Matching}

The model follows the strategy from Spatial Pyramid Matching. By using the multi-scale local spatial structure, a local pyramid hash matching algorithm is developed for similarity measurement. For example, we partition an image $I_{i}$ into $2^{l} \times 2^{l}$ segments in different levels $l=0,1, . ., L$, a total of $K=\sum 2^{l} \times 2^{l}$ bins. SPDH representation of an image $I_{i}$ is the binary code $H_{l}^{i}=\left\{H_{1}^{i}, \ldots, H_{K_{l}}^{i}\right\}$ of length $K$. In order to compare two images $I_{i}$ and $I_{j}$, 
following the work in [28], we can compute their Hamming distance for each level, and build a similarity measure upon them. The Hamming distance can be computed as:

$$
\mathcal{L}\left(H_{l}^{i}, H_{l}^{j}\right)=\sum_{k=0}^{K} D_{\text {Hamming }}\left(H_{k}^{i}, H_{k}^{j}\right)
$$

However, we cannot directly apply this method to compute the distance. For two images, the two segments $H_{k}^{i}$ and $H_{k}^{j}$ may not correspond one to the other. For example, different point of view, different scale or rotation, the spatial arrangement maybe lost or disorder. To overcome this issue, we first need to reorganize hash codes to find an optimal matching of one segment to the other. In this method, we rely on the Hungarian algorithm to minimize the discrete transport cost between the two sets of codes. First, we need to calculate the correlation between each bin in two images and organize into a similarity matrix. The similarity matrix $C^{i, j}$ between $I_{i}$ and $I_{j}$ can be defined as:

$$
C^{i, j}=\left[\begin{array}{ccc}
d_{1,1}^{i, j} & \ldots & d_{1, K}^{i, j} \\
& \vdots & \\
d_{K, 1}^{i, j} & \ldots & d_{K, K}^{i, j}
\end{array}\right]
$$

where $d_{k, r}^{i, j}$ is the Jaccard Coefficient between $H_{k}^{i}$ and $H_{r}^{j}$ :

$$
d_{k, r}^{i, j}=H_{k}^{i} \cap H_{r}^{j} / H_{k}^{i} \cup H_{r}^{j}
$$

Second, the Hungarian algorithm finds the minimum cost assignment by associating each binary codes of segment $H_{k}^{i}$ in the image $I_{i}$ to corresponding $k^{\prime}=f_{i}(k, r)$ of $H_{r}^{j}$ in the image $I_{j}$, thus the pairs of segments to be compared are identified. Finally, we can directly calculate the similarity for SPDH:

$$
D\left(H^{i}, H^{j}\right)=\sum_{k=0}^{K} d_{k, k^{\prime}}^{i, j}
$$

Figure 3 shows the proposed spatial pyramid binary pattern matching algorithm. This algorithm can take advantage of the spatial pyramid structure in SPDH, and match the duplicate regions efficiently. In experiments, this method not only achieves higher precision and recall compared with other methods, but also has the ability to capture the partial local similarity.

\section{Experiments}

In this section, we illustrate our experimental settings and results. 


\subsection{Data Sets}

We have evaluated this proposed methods on three widely used data sets, the CIFAR-10 [29], the SIVAL (Spatially Independent, Variably Area, and Lighting) dataset [30] and the Oxford buildings dataset [31]. Each dataset is split into a training set and a query set. The training set is used to train or fine-tune the CNNs network. The query set is used to evaluate the performance.

The data set CIFAR-10 consists of $60 \mathrm{~K} 32 \times 32$ images from 10 classes. Each class contains $6 \mathrm{~K}$ images. We randomly sample $1 \mathrm{~K}$ query images and use the rest as the training set.

The SIVAL dataset is a benchmark dataset that emphasizes the task of object-based image retrieval. It consists of 25 different categories with 60 images for each category. The categories consist of images of single objects photographed against highly diverse backgrounds. The objects may occur anywhere spatially in the image and also may be photographed at a wideangle or close up.

The Oxford buildings dataset consists of 5062 images collected from Flickr by searching for particular Oxford landmarks and additional 100k distractor images. The annotation provides whether an image in the database is a partial duplication of a query image for 55 queries.

\subsection{Network and Parameter Setting}

We adopt the architecture of [4] as the basic framework and resize the images so that the smaller dimension is 256. We use a 3-level pyramid $\{4 \times 4,2 \times 2,1 \times 1\}$, totally 21 bins and denoted as SPDH. In the networks, the fully-connected layer after SPP layer is replaced with the locallyconnected layer. The number of outputs of the locally-connected layer for each bin is $\{8,16,32,64\}$.

\subsection{Evaluation Protocols and Baselines}

To evaluate the retrieval performance, we compare our methods with six state-of-the-art hashing methods: supervised KSH [32], BRE [33], CNNH [24] and unsupervised LSH [34], SH [18] and IsoHash [35].

We evaluate the retrieval procedure by using Hamming ranking for comparison method. The Hamming ranking gives the ranking list for all the images in the database based on their Hamming distance or Hamming affinity to the query, where the most similar neighbors are expected to be returned 


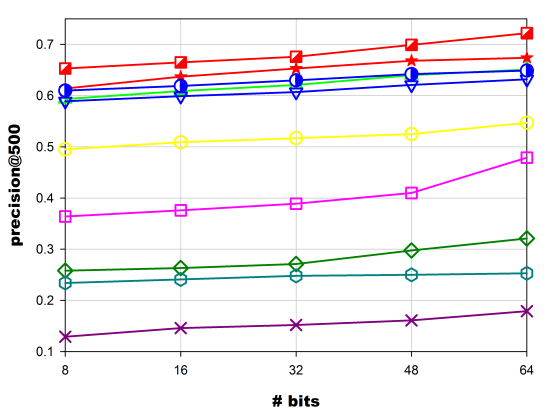

(a)

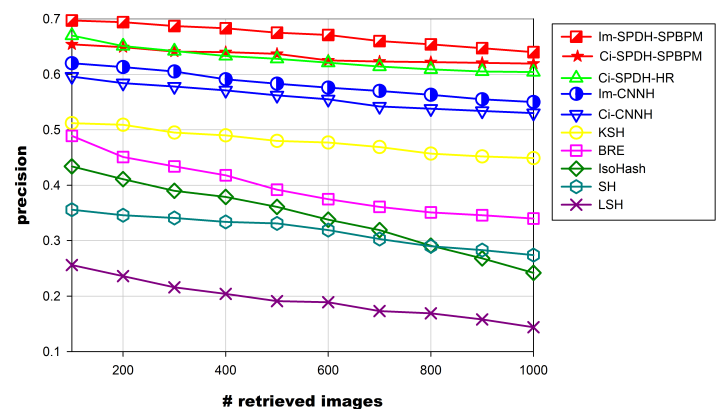

(b)

Figure 4: The results on the CIFAR-10 dataset. (a) Averaged Precision with top 500 returned vs. the different bits of hash codes; (b) Precision vs. the top retrieved images using 64-bit codes.

on the top of the ranking list. In our experiments, we also compare the Hamming ranking and local pyramid matching method introduced in sub section 3.2, respectively named as SPDH-HR and SPDH-SPBPM.

\subsection{Experiment Result on CIFAR-10}

To train and fine-tune our model to the CIFAR-10, we modify $F_{8}$ to 10 -way softmax to predict 10 object categories. We set the length of locallyconnected layer for each bin from 8 to 64 . Correspondingly the length of hash codes for SPDH and compared methods is from $8 \times 21$ to $64 \times 21$ bit. To compare with the traditional hashing learning approach with handcrafted representation, 512-d GIST features are extracted from each image. To evaluate the performance improvement by the pre-trained AlexNet for our method, we used the CIFAR-10 (Ci-SPDH) and ImageNet (Im-SPDH) as our pre-training database. Figure 4(a) shows the precision of top 500 returned image with different lengths of hash codes. Figure 4(b) reports the precision curves with different number of the top retrieved results. The MAP results are listed in Table 1. It can be seen that method achieves better performance than other supervised and unsupervised methods under the same training set (CIFAR-10). In addition, the hash learning method based on CNN can get a greater performance through pre-training on a larger dataset (ImageNet). We have also investigated more details for ten categories of CIFAR-10 dataset (Figure 5), where our method consistently outperforms all the other methods in all categories. These results suggest that using the CNN features with spatial pyramid structure can obtain high discriminative 


\begin{tabular}{|c|ccccc|}
\hline \multirow{2}{*}{ Method } & \multicolumn{5}{|c|}{ CIFAR-10(MAP \%) } \\
\cline { 2 - 6 } & 8 bits & 16 bits & 32 bits & 48 bits & 64 bits \\
\hline Im-SPDH-SPBPM & 65.71 & 66.25 & 67.12 & 67.55 & 68.01 \\
Ci-SPDH-SPBPM & 62.43 & 62.88 & 63.05 & 63.26 & 64.06 \\
Ci-SPDH-HR & 61.58 & 61.79 & 62.11 & 62.45 & 63.12 \\
Im-CNNH & 56.15 & 58.97 & 60.51 & 61.23 & 62.88 \\
Ci-CNNH & 54.81 & 58.77 & 59.87 & 60.11 & 60.26 \\
KSH & 38.64 & 41.03 & 41.78 & 42.07 & 42.22 \\
BRE & 17.44 & 19.22 & 19.88 & 20.43 & 20.51 \\
IsoHash & 21.44 & 22.44 & 24.87 & 25.07 & 25.19 \\
SH & 18.76 & 19.63 & 19.87 & 19.99 & 20.06 \\
LSH & 14.53 & 15.66 & 16.21 & 16.44 & 16.51 \\
\hline
\end{tabular}

Table 1: Image retrieval results (Mean Average Precision) with various number of bits on the CIFAR-10 dataset.

and strong spatial structure hash codes. However, by using the SPBPM for search procedure, the achieved performance gain is not significant. This is because the image of CIFAR-10 always put the object as a whole image. It has almost no partial similar images like Figure 1.

\subsection{Experiment Result on SIVAL dataset}

To evaluate the performance of the locally-connected layer in pyramid structure and SPDH method, the SIVAL dataset is used. SIVAL emphasizes the task of Localized CBIR through nearly identical scenes that varing the localized target objects only. It ensures there is one and only target object in each image. For each object class the same physical object is used in all scenes. It is also a difficult data set in that the scenes are highly diverse and often complex. Furthermore, objects may occur anywhere spatially in the image and also may be photographed at a wide-angle or close up or with different orientations. In most images, the target object occupies less than $10 \%-15 \%$ of the image area but may occupy as much as $70 \%$ in other images. Finally, the objects themselves are complex and generally correspond to multiple regions in a segmented image. We compare the retrieval results obtained from different integrating on our methods. SPDH-SPBPM: Spatial pyramid deep hashing based on the locally-connected layer and using SPBPM for image similarity computation. SPDH-HR: Hamming ranking for our framework. fcSPDH-HR: Replace the locally-connected layer with 


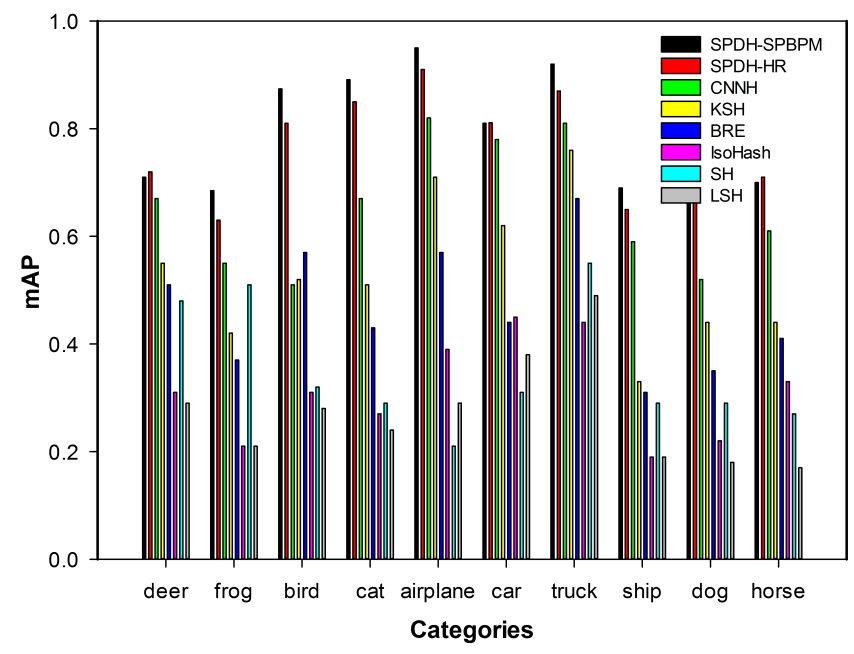

Figure 5: mAP for 10 categories in CIFAR-10

a fully-connected layer. For fair comparison, our method and CNNH will use the pre-trained model on ImageNet in parameter initialization and the 4096 dimensional CNN features are extracted from pre-trained AlexNet as the input features for other methods. Figure 6 shows the precision regarding to various number of the top images retrieved from different integration and compared methods. The proposed method SPDH-SPBPM performs more favorably against the SPDH-HR and fcSPDH-HR. Apparently, it is mainly due to the locally-connected layer and partial pyramid matching method. Because the spatial pyramid structure produced from SPP-net can be well preserved for hash codes by the locally-connected layer. On the other hand, SPBPM can use the spatial structure to match similar local regions and calculate the similarity of image pairs. Collaboration of SPDH and SPBPM show better ability for the spatially independent, variable area and lighting dataset with highly diverse backgrounds. Because other methods only generate hash codes for the entire image, it is hard to get a good precision on the SIVAL dataset. Figure 7 shows the top images retrieved by different methods. One can easily find that similar objects may occur anywhere spatially in the images with different lighting conditions and backgrounds. Our SPDH-SPBPM can successfully match the similar regions from the SIVAL database. 


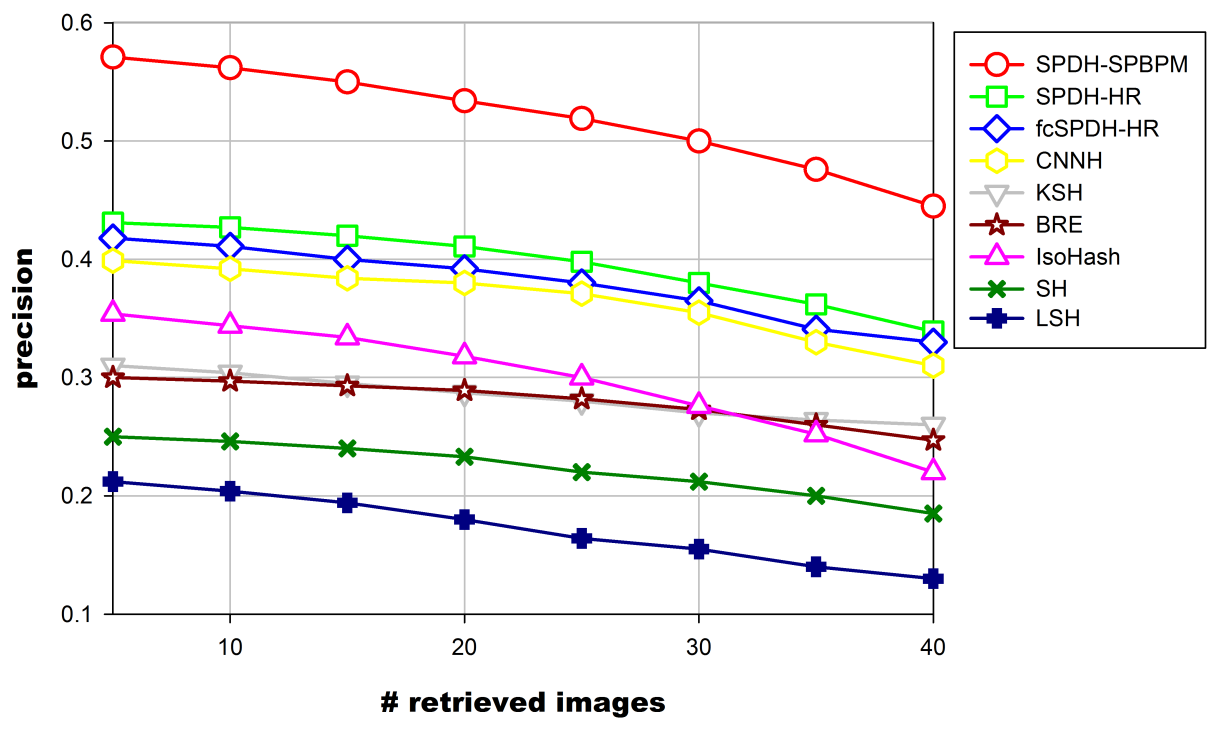

Figure 6: The precision vs. the top retrieved images using $64 \times 21$ bit codes on SIVAL dataset.

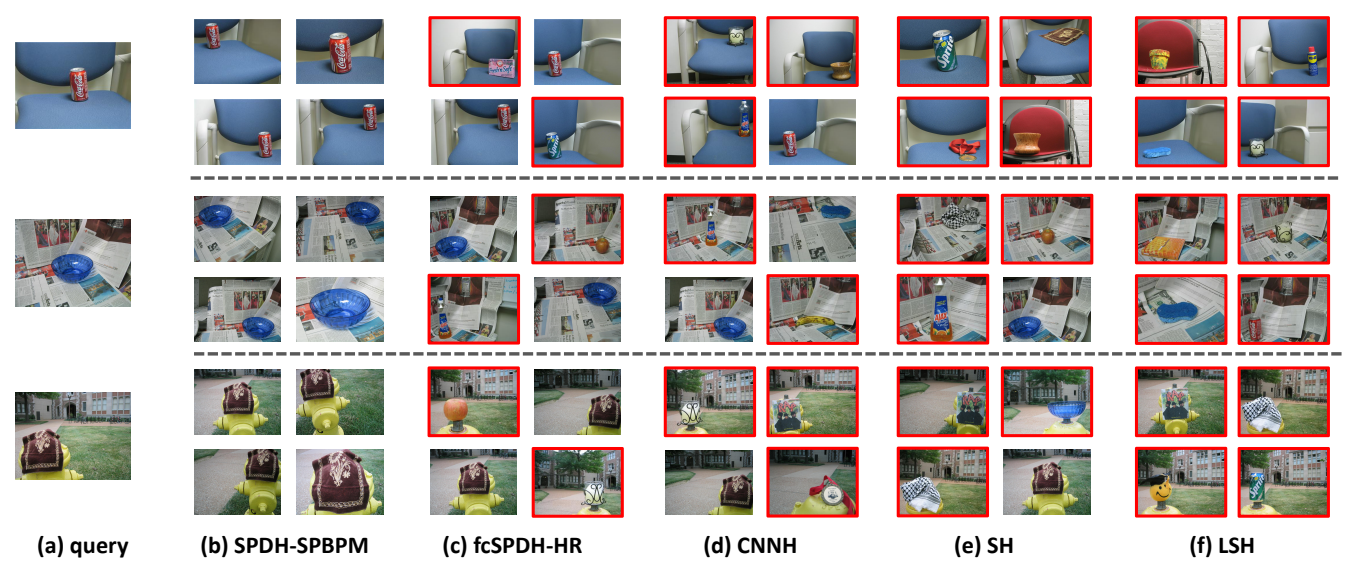

Figure 7: Top retrieved images on SIVAL dataset using $64 \times 21$ bit codes. Red border denotes false positive. 


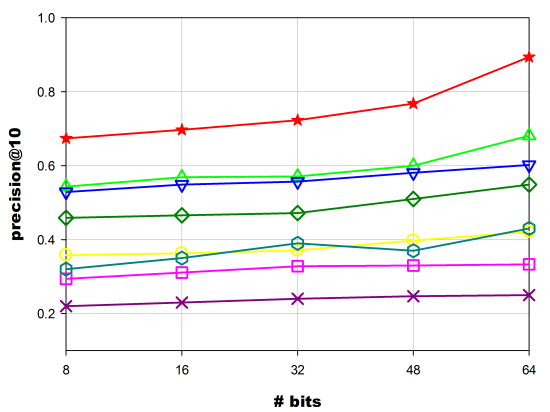

(a)

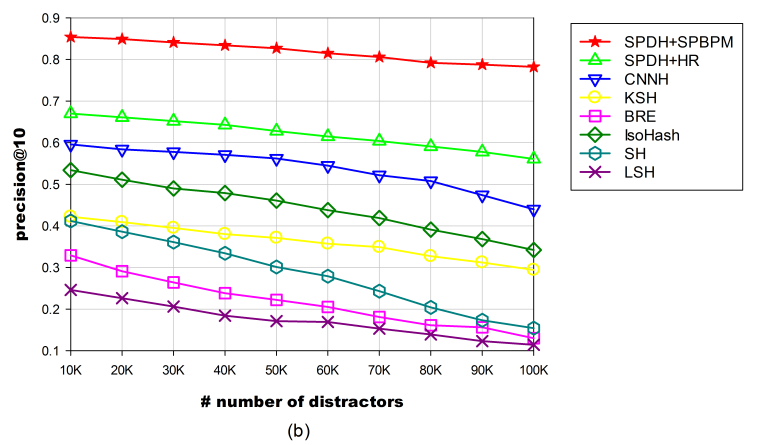

(b)

Figure 8: The results on the Oxford buildings dataset. (a) Precision curves with top 10 returned; (b) Precision curves with different numbers of distractor images.

\subsection{Experiment Result on Oxford buildings dataset}

To evaluate time consumption, we choose the Oxford buildings dataset comprising 11 different oxford "landmarks" with additional 100K distractor images. We manually labeled the 11 landmarks and non-landmark, a total of 12 classes for training. In order to be more suitable for Oxford buildings dataset, following the pervious studies [31], we construct the BoW feature by using a codebook with vocabulary size of $100 \mathrm{~K}$ for traditional hashing learning method. Similar to experiment on CIFAR-10, each bin is set from 8 to 64 in locally-connected layer, a total length is from $8 \times 21$ to $64 \times 21$ bit for SPDH and compared methods. The retrieval results for the Oxford buildings dataset are presented in Figure 8. In particular, unlike with the result of CIFAR-10 dataset, SPDH-SPBPM owns at least 20\% growth over traditional hash, which demonstrates the benefit of preserving spatial pyramid structure for spatial match. At last, Figure 9 shows example queries retrieved from Oxford buildings dataset by SPBPM with 64-bit binary codes. The results demonstrate the ability of our method to handle considerable variations in viewpoint, scale, lighting and partial occlusion from the images database.

In our framework, extracting SPDH hash codes takes around 48 milliseconds (ms) on the machine with Tesla K20m GPU and 4GB memory. Benefiting from the binary hash codes, our SPBPM only takes $0.381 \mathrm{~ms}$ on 2.1 $\mathrm{GHz}$ CPU Linux machine to compute similarity between two $64 \times 21$ bits hash codes. The average testing time and test error of our approach and competing methods on Oxford buildings dataset are reported in Table 2. For the experiments, we assume every image in the database has already been represented by the binary hash code. In this way, the time consumption of 


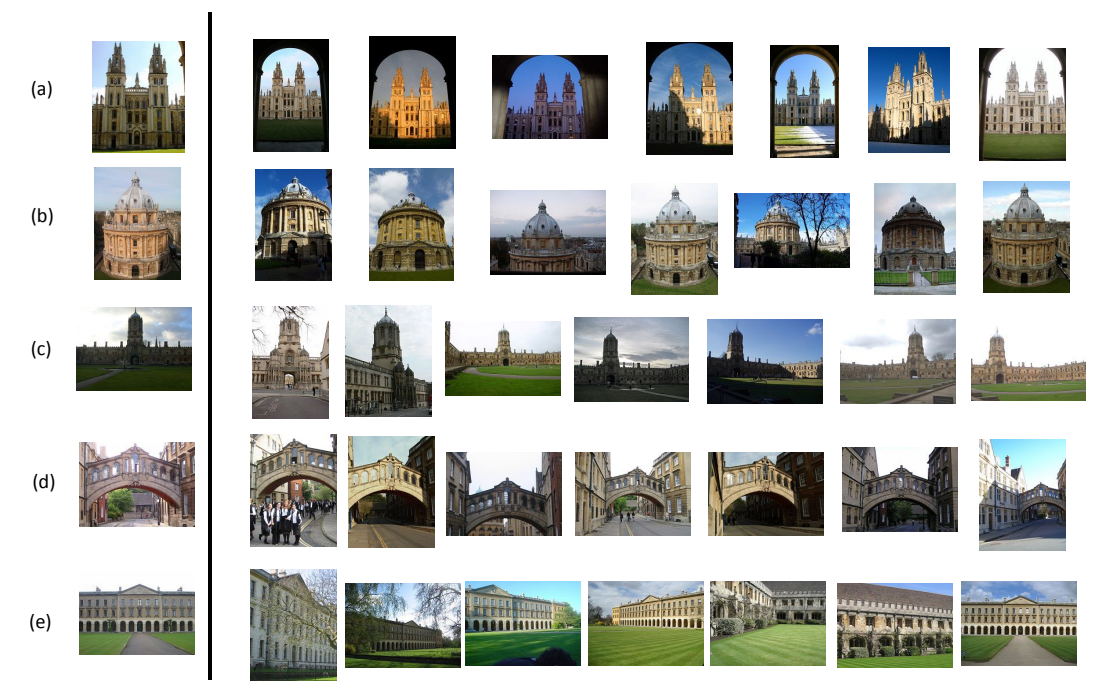

Figure 9: Examples of searching the Oxford buildings dataset for: (a) All Soul's College. (b) The Bodleian Library. (c) Christ Church. (d) Bridge of sighs, Hertford College. (e) Magdalen College. The query is shown on the left, with selected top ranked retrieved images shown to the right.

feature extraction, hash code generation and Hamming similarity computation are mainly caused by the query image. From the experimental result, our SPDH-SPBPM algorithm can significantly reduce the error rate and control the time consumption in acceptable range.

\section{Conclusions}

This paper proposes a novel deep hashing method that is able to preserve the spatial pyramid structure in binary hash codes for large scale image retrieval. The fully-connected layer $F_{7}$ of SPP-net is replaced by a locallyconnected layer to simultaneously learn domain specific image representations and a set of hash-like functions with the spatial pyramid structure. A partial similarity comparison algorithm is proposed to find partial duplicate regions in two images. Experimental results on real data sets verify greatly improvement of retrieval quality by using the deep CNNs and blending spatial information into the hash codes. In our future work, we will investigate more advanced deep learning techniques and evaluate other diverse datasets 


\begin{tabular}{|c|cc|}
\hline \multirow{2}{*}{ Method } & \multicolumn{2}{|c|}{ Oxford buildings } \\
\cline { 2 - 3 } & Average testing time(ms) & Test Error \\
\hline SPDH-SPBPM & 362.43 & 0.17 \\
SPDH-HR & 161.58 & 0.31 \\
CNNH & 154.81 & 0.39 \\
KSH & 138.64 & 0.57 \\
BRE & 117.44 & 0.62 \\
IsoHash & 121.44 & 0.45 \\
SH & 188.76 & 0.58 \\
LSH & 94.53 & 0.75 \\
\hline
\end{tabular}

Table 2: Comparison of the average testing time(millisecond per image) and test error on Oxford buildings database by fixing the code length $64 \times 21$

for more empirical studies, so that more insights can be discovered for bridging the semantic gap of multimedia information retrieval in the long term.

\section{Acknowledgment}

This research is partly supported by National Science Foundation of China under Grant 61272285, National High-Technology Program of China (863 Program, Grant No.2014AA015201), Program for Changjiang Scholars and Innovative Research Team in University (No.IRT13090), and Program of Shaanxi Province Innovative Research Team (No.2014KCT-17).

\section{References}

[1] H. Bay, T. Tuytelaars, L. V. Gool, Surf: Speeded up robust features., Computer Vision \& Image Understanding 110 (3) (2006) 404-417.

[2] D. G. Lowe, Distinctive image features from scale-invariant keypoints, International Journal of Computer Vision 60 (60) (2004) 91-110.

[3] G. Qiu, Indexing chromatic and achromatic patterns for content-based colour image retrieval, Pattern Recognition 35 (8) (2002) 1675-1686.

[4] A. Krizhevsky, I. Sutskever, G. E. Hinton, Imagenet classification with deep convolutional neural networks, Advances in Neural Information Processing Systems 25 (2) (2012) 2012. 
[5] R. Girshick, J. Donahue, T. Darrell, J. Malik, Rich feature hierarchies for accurate object detection and semantic segmentation, Computer Science (2013) 580-587.

[6] M. Oquab, L. Bottou, I. Laptev, J. Sivic, Learning and transferring mid-level image representations using convolutional neural networks, in: Computer Vision and Pattern Recognition, 2014, pp. 1717-1724.

[7] Y. Yuan, L. Mou, X. Lu, Scene recognition by manifold regularized deep learning architecture, IEEE Transactions on Neural Networks \& Learning Systems 26 (10) (2015) 1.

[8] J. Sivic, A. Zisserman, Video google: A text retrieval approach to object matching in videos, in: Computer Vision, 2003. Proceedings. Ninth IEEE International Conference on, IEEE, 2003, pp. 1470-1477.

[9] J. Yang, K. Yu, Y. Gong, T. Huang, Linear spatial pyramid matching using sparse coding for image classification, in: Computer Vision and Pattern Recognition, 2009. CVPR 2009. IEEE Conference on, IEEE, 2009, pp. 1794-1801.

[10] J. Wang, J. Yang, K. Yu, F. Lv, T. Huang, Y. Gong, Localityconstrained linear coding for image classification, in: Computer Vision and Pattern Recognition (CVPR), 2010 IEEE Conference on, IEEE, 2010, pp. 3360-3367.

[11] X. Li, L. Mou, X. Lu, Scene parsing from an map perspective., IEEE Transactions on Cybernetics 45 (9) (2015) 1.

[12] X. Lu, X. Li, L. Mou, Semi-supervised multitask learning for scene recognition., IEEE Transactions on Cybernetics 45 (9) (2015) 1.

[13] K. He, X. Zhang, S. Ren, J. Sun, Spatial pyramid pooling in deep convolutional networks for visual recognition, IEEE Transactions on Pattern Analysis \& Machine Intelligence 8691 (1) (2015) 1904-1916.

[14] M. Muja, D. G. Lowe, Fast approximate nearest neighbors with automatic algorithm configuration., VISAPP (1) 2.

[15] A. Gionis, P. Indyk, R. Motwani, et al., Similarity search in high dimensions via hashing, in: VLDB, Vol. 99, 1999, pp. 518-529. 
[16] M. Raginsky, S. Lazebnik, Locality-sensitive binary codes from shiftinvariant kernels, in: Advances in neural information processing systems, 2009, pp. 1509-1517.

[17] O. Chum, J. Philbin, A. Zisserman, et al., Near duplicate image detection: min-hash and tf-idf weighting., in: BMVC, Vol. 810, 2008, pp. 812-815.

[18] Y. Weiss, A. Torralba, R. Fergus, Spectral hashing, in: Advances in neural information processing systems, 2009, pp. 1753-1760.

[19] P. Jain, B. Kulis, K. Grauman, Fast image search for learned metrics, in: Computer Vision and Pattern Recognition, 2008. CVPR 2008. IEEE Conference on, IEEE, 2008, pp. 1-8.

[20] Y. Mu, J. Shen, S. Yan, Weakly-supervised hashing in kernel space, in: Computer Vision and Pattern Recognition (CVPR), 2010 IEEE Conference on, IEEE, 2010, pp. 3344-3351.

[21] J. Wang, S. Kumar, S. F. Chang, Semi-supervised hashing for large-scale search, Pattern Analysis and Machine Intelligence IEEE Transactions on 34 (12) (2012) 2393-2406.

[22] M. Norouzi, D. M. Blei, R. R. Salakhutdinov, Hamming distance metric learning, in: Advances in neural information processing systems, 2012, pp. 1061-1069.

[23] J. Donahue, Y. Jia, O. Vinyals, J. Hoffman, N. Zhang, E. Tzeng, T. Darrell, Decaf: A deep convolutional activation feature for generic visual recognition, University of California Berkeley Brigham Young University (2013) 647-655.

[24] R. Xia, Y. Pan, H. Lai, C. Liu, S. Yan, Supervised hashing for image retrieval via image representation learning., in: AAAI, Vol. 1, 2014, p. 2.

[25] H. Lai, Y. Pan, Y. Liu, S. Yan, Simultaneous feature learning and hash coding with deep neural networks, in: Computer Vision and Pattern Recognition, 2015, pp. 3270-3278.

[26] K. Lin, H. F. Yang, J. H. Hsiao, C. S. Chen, Deep learning of binary hash codes for fast image retrieval, in: IEEE Conference on Computer Vision and Pattern Recognition Workshops, 2015, pp. 27-35. 
[27] J. Deng, W. Dong, R. Socher, L.-J. Li, K. Li, L. Fei-Fei, Imagenet: A large-scale hierarchical image database, in: Computer Vision and Pattern Recognition, 2009. CVPR 2009. IEEE Conference on, IEEE, 2009, pp. 248-255.

[28] S. Lazebnik, C. Schmid, J. Ponce, Beyond bags of features: Spatial pyramid matching for recognizing natural scene categories, in: Computer Vision and Pattern Recognition, 2006 IEEE Computer Society Conference on, Vol. 2, IEEE, 2006, pp. 2169-2178.

[29] A. Krizhevsky, Learning multiple layers of features from tiny images.

[30] R. Rahmani, S. A. Goldman, H. Zhang, S. R. Cholleti, J. E. Fritts, Localized content-based image retrieval., IEEE Transactions on Pattern Analysis \& Machine Intelligence 30 (11) (2008) 1902-1912.

[31] J. Philbin, O. Chum, M. Isard, J. Sivic, Object retrieval with large vocabularies and fast spatial matching (2007) 1-8.

[32] W. Liu, J. Wang, R. Ji, Y.-G. Jiang, S.-F. Chang, Supervised hashing with kernels, in: Computer Vision and Pattern Recognition (CVPR), 2012 IEEE Conference on, IEEE, 2012, pp. 2074-2081.

[33] B. Kulis, T. Darrell, Learning to hash with binary reconstructive embeddings, in: Advances in neural information processing systems, 2009, pp. $1042-1050$.

[34] A. Andoni, P. Indyk, Near-optimal hashing algorithms for approximate nearest neighbor in high dimensions, in: Foundations of Computer Science, 2006. FOCS'06. 47th Annual IEEE Symposium on, IEEE, 2006, pp. $459-468$.

[35] W. Kong, W.-J. Li, Isotropic hashing, in: Advances in Neural Information Processing Systems, 2012, pp. 1646-1654. 